\title{
Der Basler Medizinprofessor Johann Jacob Harder (1656-1711) als Geburtshelfer
}

Von Marie-Louise Portmann

I

Die neue Epoche in der Geburtshilfe geht von Vesal aus, der in seinem Werk über den menschlichen Körper auch auf die weiblichen Organe, wie z. B. das Becken, eingeht. Im Anschluß an Soran hatte man nämlich noch immer an ein Auseinanderklaffen des Beckens während der Geburt geglaubt; Vesal erarbeitete eine neue Anatomie ${ }^{1}$.

Noch immer galt es als unschicklich, wenn Männer sich am Gebärbett betätigten. Dieses Feld wurde noch im 16. Jahrhundert ausschließlich von den Hebammen beherrscht. Der Zürcher Wundarzt Jakob Ruff (1500-1578), der den Auftrag hatte, das Hebammenwesen zu ordnen, war einer der ersten, der sich auf diesem Gebiet einen Namen machte. Er hielt Kurse in Geburtshilfe und verfaßte 1554 «Ein schön lustig Trostbüchle von den enpfengknussen und geburten der menschen ...» Er konstruierte auch eine Art Zange, die jedoch lediglich zur Extraktion toter Föten verwendet wurde ${ }^{2}$.

Die Geburtshilfe wurde dann vor allem in Frankreich erneuert, wo in die Gebäranstalten etwa um 1650 auch Chirurgen zugelassen wurden. Einen bedeutenden Einfluß auf die Geburtshilfe hatte vornehmlich Ambroise Paré (1510-1590), welcher selbst geburtshilfliche Operationen vornahm. Er war es, der die Wendung auf die Füße wieder einführte. In einer Schrift von 1550 , die für die Hebammen bestimmt war, kommt er eingehend darauf zu sprechen ${ }^{3}$.

Die Hebammen wurden schon früh von Chirurgen unterrichtet. Besonders berühmt ist Louise Bourgeois (um 1565 bis ca. 1645), die eine Schrift verfaßte «Observations diverses sur la stérilité, perte de fruit, fœcondite, accouchements, et maladies des femmes, et enfants nouveaux naiz». ${ }^{4} \mathrm{Sie}$ berichtet darin erstmals von der Gesichtslage und dem Nabelschnurvorfall und spricht von der abwartenden Haltung in der Nachgeburtsperiode. Im Jahre 1630 entstand im Pariser Hôtel-Dieu eine Gebärabteilung, wo dann der berühmteste französische Geburtshelfer, François Mauriceau (1637-1709), wirkte. Im Jahre 1668 veröffentlichte er die Schrift «Les 
maladies des femmes grosses et accouchées avec la bonne et véritable méthode de les bien aider», und im Jahre 1695 folgten seine geburtshilflichen Beobachtungen «Observations sur la grossesse et l'accouchement des femmes et sur leurs maladies, et celles des enfans nouveau-nés». Von Mauriceau stammen zahlreiche geburtshilfliche Fachausdrücke. Er befaßte sich auch mit pathologischen Geburten und gab Richtlinien für Komplikationen. Auch empfahl er speziell die Wendung auf die Füße. Das Auseinanderklaffen der Beckenknochen während der Geburt widerlegte er endgültig.

Grundlegend für das anatomisch-pysiologische Wissen über den Geburtsvorgang waren auch die Arbeiten William Harveys, der in seiner Schrift von 1628 den Plazentarkreislauf beschrieb. Sehr anregend wirkten auch die Forschungen des Holländers Regner de Graaf (1641-1673), der den Eierstockfollikel beschrieb. Mit der pathologischen Geburt befaßte sich der niederländische Arzt Hendrik van Deventer (1651-1724). In seinem Werk «Operationes chirurgicae novum lumen exhibentes obstetricantibus» legt er die Lehre vom engen Becken dar. Er weist darauf hin, welche Bedeutung dem Beckenraum zukommt, und bespricht die verschiedenen Beckenanomalien, die den Ablauf der Geburt erschweren ${ }^{5}$.

Im 17. Jahrhundert wurde auch zum ersten Mal die Geburtszange erwähnt. Die englische Familie Chamberlen hatte ein zangenförmiges Instrument im Gebrauch, das aber als Familiengeheimnis streng gehütet wurde. Der belgische Geburtshelfer Jean Palfyn (1650-1730) benutzte ebenfalls ein derartiges Instrument, das er unabhängig von den Mitgliedern der Familie Chamberlen erfunden hat.

Auch in der Schweiz erlebte die Entbindungskunst zu Ende des 17. Jahrhunderts einen Aufschwung. Als der bekannteste Geburtshelfer jener Zeit darf wohl der Zürcher Arzt Johann von Muralt (1645-1733) angesprochen werden. Auf seinen Studienreisen hielt er sich längere Zeit in Paris auf, wo er die Vorlesungen Mauriceaus hörte. In den Gebäranstalten arbeitete er sich, oft selbst operierend, in die Hebammenkunst ein. Nach seiner Rückkehr in die Schweiz promovierte er 1671 in Basel mit einer Dissertation über die Krankheiten der Gebärenden und die Zufälle nach der Geburt ${ }^{6}$. In Zürich war er der Lehrer vieler Schweizer Ärzte, unter anderen hörte ihn dort auch der Basler Arzt Theodor Zwinger III (1658-1724). Von Muralt scheint auch Hebammen unterrichtet zu haben, jedenfalls stand er in Beziehung zu der bekannten und erfolgreichen St. Galler Geburtshelferin Cleophea Schobinger. Diese, eine Frau von vornehmem Stand, ließ sich durch den St.Galler Arzt Tobias Baumgartner in Geburtshilfe unterrichten. Er übersetzte ihr die 
Schriften Mauriceaus ins Deutsche, und auch die Werke Cosme Viardels zog Cleophea Schobinger zu Rate ${ }^{7}$. Sie ließ Frauenleichen sezieren, um sich gynäkologische Kenntnisse zu verschaffen, damit sie für die Gebärenden keinen schädlichen Irrtum begehe. Frau Schobinger stand in engem Kontakt mit den Schaffhauser Ärzten, denen sie Krankengeschichten mitzuteilen pflegte, denn sie war besonders geschickt in der Extraktion toter Föten. Wie aus den Briefen des Schaffhauser Arztes Christoph Harder an Theodor Zwinger hervorgeht, scheint zwischen den Geburtshelfern, die Ärzte waren, und ihr eine Art Rivalität entstanden zu sein. Christoph Harder spricht vom Berufsneid des St.Galler Arztes Baumgartner, und auch von Muralt sei mißgünstig, weil sie dieselben Operationen vornehme wie $\mathrm{er}^{8}$.

Bevor die Ärzte Johann Jacob Harder und Theodor Zwinger in Basel praktizierten, betätigte sich außer den Hebammen in der Entbindungskunst hauptsächlich der Chirurg Johannes Fatio ${ }^{9}$. Ihm soll die Trennung von an Brustbein und Nabel verwachsenen Zwillingen gelungen sein. 60 Jahre nach seinem Tode erschien ein von ihm verfaßtes Hebammenbuch «Helvetischvernünftige Wehe-Mutter» 1752.

Mit Geburtshilfe beschäftigte sich auch der Arzt Franz Platter (1645-1711), der in seinen «Mantissa observationum» von 1680 schwere Geburten schildert, bei denen er selbst Hand anlegte ${ }^{10}$. Daß Theodor Zwinger geburtshilfliche Praxis trieb, wissen wir aus seinen Briefen.

II

Wenden wir uns nun Johann Jacob Harder zu, der in Basel, Straßburg, Genf, Lyon und Paris studierte und 1678 in seiner Heimatstadt Professor wurde ${ }^{11}$. Über seine Tätigkeit als Geburtshelfer berichtet er in seinem «Apiarium observationibus medicis et experimentis refertum» (Basel 1687). Er beschäftigte sich darin eingehend mit embryologischen und gynäkologischen Fragen und baute dabei hauptsächlich auf den Forschungen des Fabricius ab Aquapendente auf (1547-1619), der ein Schüler des Fallopius war. Fallopius (1523-1562) seinerseits war ein Schüler Vesals und hatte in Anlehnung an seinen Lehrer die weiblichen Geschlechtsorgane beschrieben. Bei Fabricius ab Aquapendente hörte auch William Harvey, dessen Beiträge zur Embryologie Harder ebenfalls kannte. Auch die Schriften des englischen Arztes Walter Needham (1631-1687) und des Holländers Nicolas Hoboken (1632-1678) zog Harder bei ${ }^{12}$. Er kannte ebenfalls die Arbeiten des Holländers de Graaf. 
Da menschliche Leichen sehr schwer zu bekommen waren und es eine Seltenheit war, den Leichnam einer schwangeren Frau sezieren zu können, übte sich Harder in der Zergliederung trächtiger Tiere. Im «Apiarium» gibt er verschiedene Sektionsbefunde an Kühen, Hasen, Kaninchen, Schweinen und Schafen wieder. Hie und da bot sich ihm jedoch Gelegenheit, den Leichnam einer Wöchnerin zu zergliedern. So berichtet er am 17. Juli 1678 in einem Brief an Johann von Muralt: «Weil der Bote gerade weggeht, füge ich nichts anderes bei, außer daß mir vor einigen Wochen eine ausgezeichnete Gelegenheit geboten wurde, die Substanz des Uterus zu untersuchen bei der Sektion des Leichnams einer Wöchnerin. Ich fand die Substanz lose, schwammig, voll von reichlichen Poren, bei deren Pressung viel Flüssigkeit herauströpfelte». ${ }^{13}$ Aus dem «Apiarium» ${ }^{14}$ erfahren wir, daß sich für Harder im Oktober 1681 anläßlich einer gerichtsmedizinischen Untersuchung Gelegenheit bot, bei der Anatomie einer schwangeren Frau mitzuwirken. Eine über 40 jährige Witwe, die in anderen Umständen war, ohne daß jemand davon wußte, starb ganz plötzlich. Es verbreitete sich sodann das Gerücht in der Stadt, sie habe Gift genommen. Der Basler Rat beauftragte hierauf den Stadtarzt Johann Rudolf Burckhardt und andere Ärzte mit der Untersuchung des Leichnams. Dabei wurden auch Harder, Johann Conrad Peyer aus Schaffhausen und ein gewisser Doktor Haesbaert zugelassen. Auch zahlreiche Chirurgen waren zugegen. Harder wirkte bei dieser Anatomie als Prosektor. Zunächst wurde der Magen untersucht, wobei die Rückstände eines weißen Pulvers festgestellt wurden. Weiter wurden die Leber, das Pankreas und die Gallenblase untersucht. Aus dem Uterus löste Harder sodann einen männlichen Foetus von fünf Monaten, den er mit nach Hause nahm und unter seinen Kuriositäten aufbewahrte. Zuletzt wurden noch die Lungen untersucht, wobei man aber nichts Besonderes feststellte, außer einer Struma am Halse. Nachforschungen ergaben folgendes: Die Hausbewohner hatten bemerkt, daß die Verstorbene ein Pulver in ihre Speise mischte. Es handelte sich um ein Purgativum, das sie schon zweimal zu sich genommen hatte und das sie zum Brechen reizte. Es wurde vermutet, daß sie mittels dieses starken Medikaments die Frucht abtreiben wollte. Der Versuch jedoch kostete ihr und dem Kinde das Leben.

Es seien nachstehend diejenigen Observationen Harders ins Licht gerückt, die in das eigentliche Gebiet der Geburtshilfe gehören. Der Basler Arzt hatte die Schriften des Franzosen Mauriceau genau studiert, denn er kommt an verschiedenen Stellen des «Apiarium» darauf zu sprechen. In der Observation LXXXIV ${ }^{15}$ berichtet er von einer Uterusblutung mit nachfol- 
gender Frühgeburt. Im Januar 1680 wurde er zu einer etwas über 30 jährigen Frau gerufen, die schon Mutter mehrerer Kinder war. Sie hatte jedoch schon einige Frühgeburten gehabt oder die Kinder waren während der Geburt gestorben. Sie war jetzt im fünften Schwangerschaftsmonat. Da sie, wie Harder meint, hypochondrisch war und zu Blutungen der Hämorrhoiden neigte, riet ihr Harder an, sich zur Ader zu lassen, um der Plethora (Vollblütigkeit) entgegenzuwirken. Diesen Eingriff nahm ein Chirurgus vor, und in der darauffolgenden Nacht trat eine Uterusblutung ein, die jedoch nicht sehr heftig war. Harder wurde gerufen, und er stellte fest, daß der Foetus eine andere Lage eingenommen hatte. Er verschrieb ein Medikament zum Einnehmen und ein Pflaster, auf den Unterleib aufzulegen. Bis zum 16. März schien alles in Ordnung zu sein, als sich plötzlich das Übel wiederholte; die Kranke klagte über heftige Schmerzen im Unterleib bis zu den Lenden. Medikamente fruchteten nichts, und am 22. März verschärften sich die Schmerzen und erstreckten sich bis zum Schambein. Harder erwähnt, daß diese Erscheinung «die schneidende Wehe» genannt werde. Der Arzt war nun im Zweifel, was er tun sollte; die Blutung schien ihm ein schlechtes Zeichen, während er andererseits, da der Foetus sich bewegte und das Kind noch nicht ausgetragen war, nicht eine Geburt für bevorstehend hielt. Er riet indessen ein Klistier an, das die Geburtswege reinigen sollte, denn bei dessen Anwendung, schreibt Harder, pflege die Natur deutlich anzuzeigen, ob eine Geburt bevorstehe. Die Kranke jedoch wollte nichts von diesem Klistier wissen, und so verschrieb ihr Harder ein Medikament und Säckchen, um den Unterleib zu erwärmen. Nach dieser Therapie entleerte sich das Fruchtwasser, worauf ein Blutfluß folgte. Der Kranken wurden indessen Stärkungsmittel und Brühen gereicht. Als wieder Wehen auftraten, vermochte die Hebamme das Kind, das etwa sieben Monate alt war, bei den Füßen herauszuziehen. Damit schließt Harder diese Beobachtung ab.

Des weiteren ${ }^{16}$ berichtet Harder den Fall einer etwa 30 jährigen Bäuerin, die im letzten Schwangerschaftsmonat am linken Oberschenkel eine Venenblutung erlitt. Durch Verbände und Heilmittel versuchte Harder, ihr zu helfen, aber zwei Wochen später brach die Wunde erneut auf, als sie allein beim Heuen war. Harder verschrieb ihr, als er gerufen wurde, ein Mittel, um die Wehen zu befördern,worauf die Geburt einen normalen Verlauf nahm. Abends besuchte der Arzt die Wöchnerin wieder, zusammen mit Doktor Schorkopf. Er verschrieb ihr ein Medikament und verordnete stärkende Brühen. Das Weintrinken verbot er ihr. Da die Kranke Harders Verordnungen Folge leistete, erlangte sie später ihre frühere Gesundheit wieder. 
An anderer Stelle ${ }^{17}$ berichtet Harder, wie er bei einer anormal verlaufenen Geburt auf die Bitte des Pfarrers hin selbst Hand anlegte. Die 38 jährige Gattin eines Schmiedes, die ihrem Gatten bei der Arbeit zu helfen pflegte, hatte sich vor der Geburt mit dem Hammer eine innere Verletzung zugezogen, dies aber verschwiegen. Als die Zeit der Schwangerschaft abgelaufen war, setzten die Wehen ein, und am 6. Mai 1681 floß das Fruchtwasser ab. Die Geburt wollte nicht vorwärts gehen, obwohl mehrere Hebammen dabei waren. Am 8. Mai wurde Harder zugezogen, und er ließ sich zunächst erzählen, was bis jetzt geschehen war. Die Hebamme war der Meinung, der Muttermund sei fest verschlossen, und schrieb es diesem Umstand zu, daß die Geburt nicht vorwärts ging. Harder vermutete gleich, daß die Frucht schon seit mehreren Stunden abgestorben sei, erstens wegen der langen Zeit, die schon verstrichen war, und zweitens, weil keine Wehen mehr auftraten. Er verordnete sogleich ein stimulierendes Clysma und auch stärkere Medizin zum Einnehmen. Trotz den Anstrengungen der Gebärenden und der Hebamme klagte diese darüber, daß noch gar nichts vom Kinde wahrzunehmen sei. Darin habe sie sich aber getäuscht, meint Harder. Denn jetzt, auf die Bitte des Pfarrers hin, stand er selbst der gefährdeten Mutter bei und stellte sogleich fest, daß der Muttermund geöffnet sei. W as die Hebamme getäuscht hatte, war die mit Blut gefüllte Haut über dem Schädel des Kindes, die wie eine mit Wasser gefüllte Blase den Ausgang versperrte, so daß die Hebamme nicht etwas Hartes, das sie als den Kopf des Kindes hätte erkennen können, sondern einen weichen Körper wahrnahm. Harder erweiterte sorgfältig den Muttermund und vergewisserte sich über die Lage des Foetus. Da Harder erkannte, daß jener weiche Körper den Weg versperre, ließ er die Gebärende zunächst bequem betten und ihr stärkende Brühen reichen. Sodann machte er mit einem Instrument einen Einschnitt, und es gelang ihm dann, zunächst den Kopf des Kindes, dessen Schädelknochen zerstört waren, und dann den ganzen Leib zu extrahieren. Auch die Placenta löste er dann vorsichtig los. Harder verordnete nach dieser Operation eine zweckmäßige Diät und auch Scheidenspülungen, die, wie er meinte, zur Reinigung und um die Schärfe der Säfte zu mildern dienen sollten. Die Kranke hatte noch einige Zeit Fieber, und der Unterleib schwoll etwas auf, jedoch am 18. Mai ging es ihr bereits besser, und sie erlangte dann die frühere Gesundheit wieder. Harder erwähnt noch, daß sie später einem gesunden Kind das Leben schenkte.

Einen Fall von Placenta praevia schildert Harder in der Observatio $\mathrm{XC}^{18}$. Eine 40 jährige Frau aus dem Solothurner Gebiet, Mutter mehrerer Kinder, befielen die Wehen am 18. Juli 1683. Zuerst entleerte sich das 
Fruchtwasser, und darauf folgte sogleich die Nachgeburt. Der Foetus jedoch gelangte nicht an die Außenwelt, obschon verschiedene Hebammen ihre Künste versuchten. Am 20. Juli begann das Kind abzusterben, was die Mutter an der Gewichtszunahme und am Fehlen jeglicher Bewegung bemerkte. Man wartete jedoch bis zum neunten Tag, bis zum 28. Juli, auf die Geburt, bis endlich der Gatte den Arzt Harder um seinen Beistand bat. Dieser rügte zunächst das lange Zuwarten und wies auf die Gefahr hin, welche die Zersetzung des Foetus für die Mutter bedeutete, verschrieb jedoch sofort Medikamente und besuchte nach einigen Stunden zusammen mit Doktor Scherb aus Bischofszell die Wöchnerin. Der Unterleib war sehr ausgedehnt, die Kranke klagte über Durst, der Puls war etwas beschleunigt, und es waren keine Anzeichen von Wehen vorhanden. Durch den üblen Geruch schloß Harder darauf, daß der Foetus bereits in Zersetzung übergegangen war, und er machte sich sofort bereit zur Operation. Von Hand eröffnete und erweiterte er den inneren Muttermund und stellte die anormale Lage des Foetus fest; die Schädelknochen des Kindes klafften auseinander. Harder zog den Foetus nach vorn, und mit einem Instrument durchschnitt er die den Schädel des Kindes umgebenden Hüllen, worauf er die Öffnung von Hand erweiterte. Wegen der anormalen Lage der Glieder kostete es ihn einige Mühe, den ganzen Leib des Kindes zu extrahieren. Harder verschrieb dann eine Diät und zur Reinigung Scheidenspülungen. Am elften Tag nach dieser Operation berichtete der Gatte dem Arzt, daß die Wöchnerin wenig Durst verspüre, Appetit habe und ohne Schwierigkeiten herumgehen könne. In der Folge erholte sie sich.

Sodann schildert Harder ${ }^{19}$, wie er am 19. Juni 1683 bei einer Zwillingsgeburt Beistand leistete. Am Tage vorher hatte die Frau ein Mädchen geboren. Da aber der Unterleib ausgedehnt blieb und sich keine Wehen mehr einstellten, wurde Harder zu Rate gezogen. Diesem gelang es dann, mittels der Wendung auf die Füße das andere Kind zur Welt zu bringen. Die Wöchnerin litt nachher noch an einer «Uterusentzündung», und Harder behandelte sie weiterhin, um die Ausdehnung des Unterleibs, die sich nach der Geburt nicht zurückgebildet hatte, zum Verschwinden zu bringen. Er verordnete einen Verband, Umschläge, Säckchen zum Auflegen und wiederum reinigende Spülungen. Zum Einnehmen verschrieb er ein schweißtreibendes Mittel. Auch hier gelang es ihm, die Patientin am Leben zu erhalten.

Aus Harders Observationen ist zu ersehen, daß dieser Basler Arzt über ein für seine Zeit angemessenes geburtshilfliches Können verfügte und daß er mit den modernsten Methoden vertraut war. Als erste Autorität auf diesem 
Gebiet galt für ihn ohne Zweifel Mauriceau, auf den er sich immer wieder beruft.

\section{Anmerkungen}

1 Vesalius, Andreas, De humani corporis Fabrica, Basel 1543, S. $131 \mathrm{f}$.

${ }^{2}$ Buess, H., und Portmann, M.-L., Médecins suisses célèbres: 61 Jakob Ruff (ca. 1500-1578). Médecine et Hygiène 37, 12 septembre 1979, p. 2944-2945.

3 Paré, Ambroise, La manière de extraire les enfans tant mors que vivans hors le ventre de la mère, lors que nature de soy ne peult venir a son effect. CEuvres complètes, vol. II, Paris 1840, p. 628.

${ }^{4}$ Paris 1609.

${ }^{5}$ Hodel, C., und Portmann, M.-L., Hendrik van Deventer (1651-1724), ein Pionier der modernen Geburtshilfe. Gynäkologische Rundschau 6, S.307-312, 1968.

${ }^{6}$ Die Matrikel der Universität Basel, Band III, Basel 1962, S.590. Husner, Fritz, Verzeichnis der Basler medizinischen Universitätsschriften von 1575-1829. In: Festschrift für Jacques Brodbeck-Sandreuter, Basel 1942, S.209. Johannes von Muralt publizierte auch ein «Kinder- und Hebammenbüchlein», Zürich 1689.

7 Viardel, Cosme, Observations sur la pratique des accouchements, Paris 1671.

${ }^{8}$ Buess, H., Portmann, M.-L., Molling, P., Theodor Zwinger III (1658-1724). Ein Basler Arzt und Kinderarzt der Barockzeit. Studien zur Geschichte der Wissenschaften in Basel, Fasc. XIV, Basel 1962, S. 52 .

${ }^{9}$ Gerster, J., Johannes Fatio, ein Basler Chirurg und Geburtshelfer des 17.Jahrhunderts. Basel 1917.

${ }^{10}$ Er gab 1680 eine neue Auflage der «Observationum libri III» seines Großonkels Felix Platter I heraus. Das Buch trägt den Titel «Observationum selectiorum a diariis practicis passim excerptarum mantissa».

11 Zur Biographie J.J. Harders siehe:

- Burckhardt, Albrecht, Geschichte der Medizinischen Fakultät zu Basel 1460-1900. Basel 1917, S. 191-195.

- Brunner, Conrad, und von Muralt, Wilhelm, Aus den Briefen hervorragender Schweizer Ärzte des 17. Jahrhunderts. Basel 1919, S.288-304.

- Joos-Renfer, S., Portmann, M.-L. und Buess, H., Pathologisch-anatomische Beobachtungen bedeutender Schweizer Ärzte 1670-1720. Basler Veröffentlichungen zur Geschichte der Medizin und der Biologie Fasc. XIII, Basel 1961, S. 19-20.

-- Die Matrikel der Universität Basel, Band IV, Basel 1975, S. 15-16.

12 Needham schrieb «Disquisitio anatomica de formato foetu», London 1667, und Hoboken, der aus Utrecht gebürtig war und später eine Professur in Steinfurt innehatte, verfaßte einen Traktat «Anatomia secundinae humanae», 1669.

13 Brunner, Conrad, und von Muralt, Wilhelm, a.a. O., S.293.

${ }^{14}$ Harder, J.J., Apiarium observationibus medicis et experimentis refertum. Basel 1687, Observatio XI. 
15 Ebenda, S. 318-319.

${ }_{16}$ Ebenda, Observatio LXXXV, S. 322.

17 Ebenda, Observatio LXXXIX, S. 333-336.

${ }^{18}$ Ebenda, S. 336-338.

${ }^{19}$ Ebenda, S. 340-341.

\section{Summary}

First an outline of obstetric progress in the 16th and 17th centuries is given and the most important Swiss accoucheurs are represented. Then the interests of Johann Jacob Harder (1656-1711) in this field are pointed out, who wrote a series of observations which he published 1687 in his «Apiarium». The author was mainly influenced by French obstetricians, especially François Mauriceau (1637-1709).

Dr. M.-L. Portmann

Medizinhistorische Bibliothek

Klingelbergstraße 23

CH-4031 Basel 